\title{
Form factors and structure functions of heavy mesons and baryons
}

\author{
Kamil Serafin* \\ Faculty of Physics, University of Warsaw, Pasteura 5, 02-093, Warsaw, Poland \\ Institute of Modern Physics, Chinese Academy of Sciences, 509 Nanchang Rd., Lanzhou 730000, \\ China \\ E-mail: kserafin@impcas.ac.cn
}

\begin{abstract}
I present preliminary results for form factors and structure functions of some of spin- 0 , spin-1/2 and spin-1 heavy quarkonia and triply-heavy baryons in heavy flavor QCD. Using renormalization group procedure for effective particles (RGPEP) and gluon mass ansatz, approximate Hamiltonians for heavy quarkonia and triply-heavy baryons in QCD were found. The approximate eigenfunctions of these Hamiltonians are used to obtain the results for form factors and structure functions. The results for charge radii are in a surprisingly good agreement with lattice QCD results in cases where comparison is possible. Magnetic moments of vector charmonia and bottomonia are in agreement with other theoretical results. Magnetic moments of $B_{c}^{*}$ mesons and spin-1/2 ground states of $b b c$ and $c c b$ baryons are considerably larger than moments reported in the literature. The hadron structure functions are computed in a simplified way, neglecting the huge difference between the scale of quark binding and the scale of the virtual photon in deep inelastic scattering.
\end{abstract}

Light Cone 2019 - QCD on the light cone: from hadrons to heavy ions - LC2019

16-20 September 2019

Ecole Polytechnique, Palaiseau, France

\footnotetext{
*Speaker.
} 


\section{Introduction}

It has been shown $[1,2]$ that renormalization group procedure for effective particles (RGPEP) together with an ansatz that effective gluons acquire mass produces in the second order in the strong coupling constant a harmonic confining potential between quarks composing mesons or baryons in heavy-flavor QCD (with charm and bottom quarks only). Effective interactions include also smeared chromoelectric Coulomb potential with Breit-Fermi terms. In order to produce a sketch of spectra of heavy mesons and baryons a crude approximation is adopted in Ref. [2]: all Breit-Fermi terms are omitted, the Coulomb potential is treated as a perturbation to the harmonic oscillator potential and the smearing is also neglected. Masses are computed analytically, but the errors can be as big as $\sim 100 \mathrm{MeV}$, which is the order of magnitude of the splitting between $J / \psi$ and $\eta_{c}$ (not reproduced in the sketch). In practice, the errors are smaller for spin-1 quarkonia states because several low-mass spin-1 states of heavy quarkonia were used to fit the free parameters (masses of quarks and renormalization-group scale parameters). Masses of ground states of triply-heavy baryons, which are then predicted, are very close to the lattice results (the differences are smaller than $30 \mathrm{MeV}$ ) with the exception of the $\Omega_{c c b}$ baryon, which is about $300 \mathrm{MeV}$ too high. For singleflavor baryons the splittings between the ground state and radially or orbitally excited states are in qualitative agreement with the lattice results. Analogous lattice results for $c c b$ and $b b c$ systems are not available at the moment. Therefore, the sketch of the meson and baryon spectra can be considered as reasonable bearing in mind the severity of the approximations, but also suggests that the gluon mass ansatz or the approach itself needs to be adjusted for mixed-flavor systems.

The computation of masses can be improved in many ways, the most important being the calculation of effective Hamiltonians in the fourth order of the strong coupling constant $g$. However, one can also perform a rather straightforward additional check of the hadron states obtained so far by calculating form factors and structure functions of heavy mesons and heavy baryons [3]. The results are presented in the next two sections. I use harmonic oscillator wave functions obtained in Ref. [2].

\section{Form factors}

Form factors of hadrons are extracted from hadron matrix elements of the electromagnetic current, $J_{\sigma^{\prime} \sigma}^{\mu}\left(P^{\prime}, P\right)=\left\langle P^{\prime}, \sigma^{\prime}\left|J^{\mu}(0)\right| P, \sigma\right\rangle$, where $P$ and $\sigma$ denote the momentum and front form spin projection of the initial hadron state, $P^{\prime}$ and $\sigma^{\prime}$ denote the momentum and front form spin projection of the final hadron state, and $J^{\mu}(0)=J^{\mu}\left(x^{\nu}=0\right)$ is the electromagnetic current operator. In all calculations I assumed that $P^{\prime+}=P^{+}$. I consider spin-0, spin-1/2 and spin-1 particles, which have one, two and three form factors, respectively [3]. Except for the spin-0 case, one cannot unambiguously extract form factors from $J_{\sigma^{\prime} \sigma}^{\mu}$ because it does not have the expected Lorentz-covariant structure. This problem is often attributed to the fact that rotations are dynamic in front form of Hamiltonian dynamics. Details of the extraction can be found in Ref. [3]. I focus only on certain characteristics of the form factors - charge radii and magnetic moments, because measurement of the form factors themselves is very difficult for heavy quarkonia, and triply-heavy baryons have not been even observed yet. To reliably compute magnetic moments I included relativistic corrections to wave functions, which improve agreement between the expected relativistically covariant 
form of $J_{\sigma^{\prime} \sigma}^{\mu}$ and the actual $J_{\sigma^{\prime} \sigma}^{\mu}$ that comes from the calculations. The corrections change the nonrelativistic spin structure of the states a little bit, but leave the radial and orbital wave functions intact. For example, for vector quarkonia the spin structure is given by $\bar{u}_{1} \gamma_{\mu} \varepsilon^{\mu} v_{2}$, which in the nonrelativistic limit gives the spin-triplet wave function as expected plus corrections of order momentum divided by mass. Finally, for charmonia and bottomonia I perform calculations as if the antiquark had no electric charge, which is the usual way of avoiding zero form factors for those neutral particles. The results are collected in tables 1,2 and 3.

\begin{tabular}{|c|c|c|c|c|c|c|c|c|c|c|}
\hline \multicolumn{6}{|c|}{$c \bar{c}$} & \multicolumn{5}{|c|}{$b \bar{b}$} \\
\hline & $\eta_{c}(1 S)$ & $\chi_{c 0}(1 P)$ & $\eta_{c}(2 S)$ & $J / \psi$ & $\psi(2 S)$ & $\eta_{b}(1 S)$ & $\chi_{b 0}(1 P)$ & $\eta_{b}(2 S)$ & $\Upsilon(1 S)$ & $\Upsilon(2 S)$ \\
\hline$r$ & 0.249 & 0.322 & 0.381 & 0.257 & 0.385 & 0.152 & 0.196 & 0.232 & 0.154 & 0.233 \\
\hline [4] & 0.207 & 0.265 & 0.386 & 0.212 & 0.387 & 0.126 & 0.192 & 0.237 & 0.126 & 0.239 \\
\hline [5] & 0.210 & & & 0.261 & & 0.110 & & & 0.195 & \\
\hline [6] & 0.251 & 0.308 & & 0.257 & & & & & & \\
\hline [7] & 0.219 & & & 0.228 & & & & & & \\
\hline \multicolumn{6}{|c|}{$c \bar{b}$} & & \multicolumn{4}{|c|}{$\Omega$} \\
\hline & $B_{c}(1 S)$ & $\chi_{b c 0}(1 P)$ & $B_{c}(2 S)$ & $B_{c}^{*}(1 S)$ & $B_{c}^{*}(2 S)$ & & $c c c$ & $c c b$ & $b b c$ & $b b b$ \\
\hline$r_{c}$ & 0.337 & 0.435 & 0.515 & 0.342 & 0.516 & & 0.31 & 0.35 & 0.32 & \\
\hline$r_{b}$ & 0.105 & 0.136 & 0.160 & 0.106 & 0.161 & & & 0.18 & 0.20 & 0.19 \\
\hline$r$ & 0.282 & 0.364 & 0.430 & 0.286 & 0.433 & & 0.31 & 0.39 & 0.20 & 0.19 \\
\hline [8] & & & & & & & 0.29 & & & \\
\hline
\end{tabular}

Table 1: Summary of charge radii of mesons and baryons. Rows with citations are the results found in literature and are presented for comparison. All other numbers are my results [3]. The number of digits that I provide is adjusted to make visible the difference between radii of vector and pseudoscalar particles. All numbers are in $\mathrm{fm}$. The references can be characterized by the method of calculation: Basis Light Front Quantization [4], Contact Interaction [5], Dyson-Schwinger equations [7], and Lattice [6, 8]. $r_{c}$ and $r_{b}$ are charge-normalized contributions from quarks $c$ and $b$.

\begin{tabular}{|c|c|c|c|c|c|}
\hline & RGPEP [3] & BLFQ [4] & CI [5] & Lattice [6] & DSE [7] \\
\hline$J / \psi$ & $\mathbf{2} \pm \mathbf{0 . 1 3}$ & $1.952(3)$ & 2.047 & $2.10(3)$ & $2.13(4)$ \\
$\psi(2 S)$ & $\mathbf{2} \pm \mathbf{0 . 5 4}$ & $2.05(2)$ & & & \\
\hline$\Upsilon(1 S)$ & $\mathbf{2} \pm \mathbf{0 . 0 2}$ & $1.985(1)$ & 2.012 & & \\
$\Upsilon(2 S)$ & $\mathbf{2} \pm \mathbf{0 . 1 4}$ & $1.992(1)$ & & & \\
\hline
\end{tabular}

Table 2: Magnetic moments $\mu$ of heavy quarkonia neglecting the contribution from antiquark. The theoretical relative uncertainty is estimated as the absolute value of binding energy over the sum of quark masses, which is the order of magnitude of the ambiguity in extraction of magnetic form factor from hadronic matrix elements $J_{\sigma^{\prime} \sigma}^{\mu}$.

\section{Structure functions}

To obtain structure functions I computed the hadronic tensor in deep inelastic unpolarized scattering. Its components $W^{\mu v}$ with $\mu, v=+, 1,2$, have the expected relativistic form with two 


\begin{tabular}{|c|c|c|c|c|c|}
\hline & Serafin [3] & Lahde [9] & Dhir [10] & Faessler [11] & Simonis [12, 13] \\
\hline$B_{c}^{+*}(1 S)$ & $\mathbf{3 . 2 5} \pm \mathbf{0 . 0 7}$ & 2.88 & & & 2.57 \\
$B_{c}^{+*}(2 S)$ & $\mathbf{3 . 2 4} \pm \mathbf{0 . 3 5}$ & 2.65 & & & \\
\hline$\Omega_{c c b}$ & $\mathbf{5 . 1 6} \pm \mathbf{0 . 4 6}$ & & $4.49,4.62$ & 4.69 & 4.03 \\
$\Omega_{b b c}$ & $-\mathbf{2 . 7 7} \pm \mathbf{0 . 0 9}$ & & $-2.45,-2.39$ & -2.39 & -2.24 \\
\hline
\end{tabular}

Table 3: Dipole magnetic moments of mixed-flavor particles. The theoretical relative uncertainty is estimated in the same way as in table 2.

structure functions $F_{1}\left(x, Q^{2}\right)$ and $F_{2}\left(x, Q^{2}\right)$, which I calculated in the Bjorken limit. From a renormalization group procedure point of view one should use two different kinds of particles (creation and annihilation operators) - one that is related to the scale of binding, which is relatively small and best suited to describe bound states, and the other related to formally infinite $\left(Q^{2} \rightarrow \infty\right)$ scale of hard electromagnetic interaction. RGPEP provides necessary tools to translate one type of particles to another, but in the present work I approximated the transformation connecting different types of particles by unity. As a result the structure functions do not depend on $Q^{2}$. Proper treatment of different types of particles should give $Q^{2}$ dependence. The results are presented in Figs. 1 and 2.
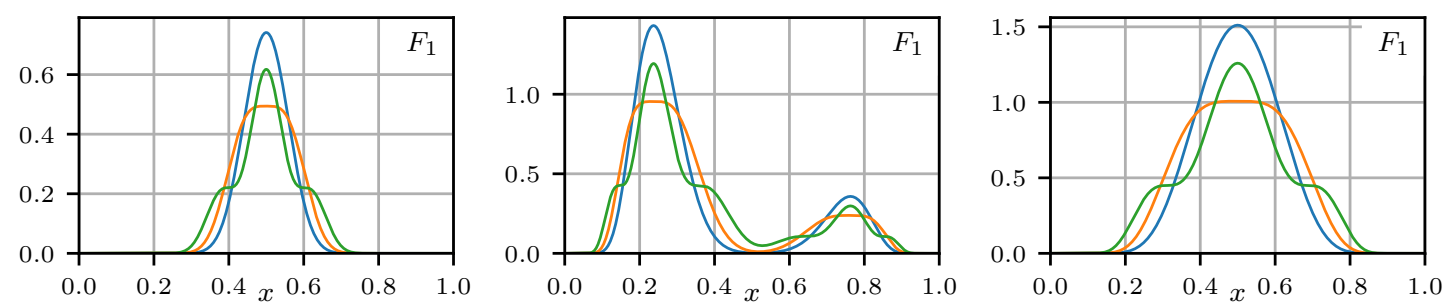

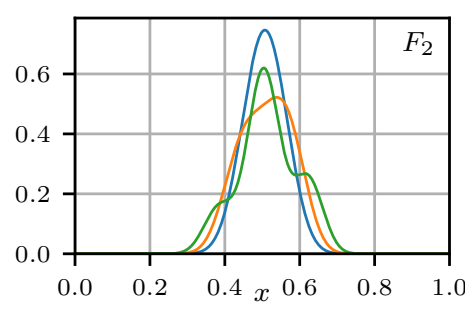

(a) Bottomonium

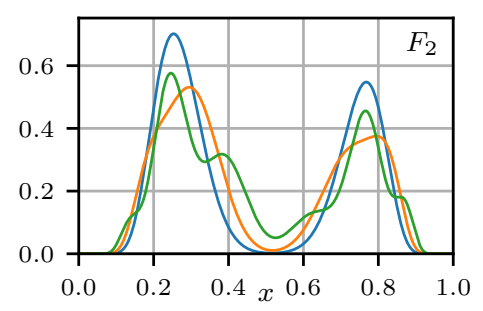

(b) $B_{c}$

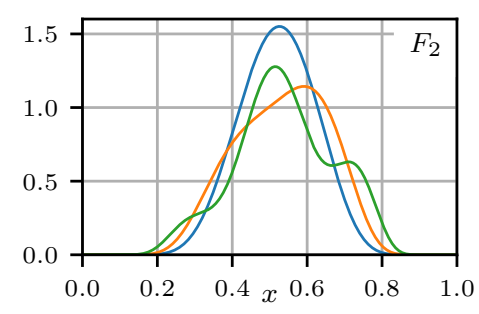

(c) Charmonium

Figure 1: Structure functions of heavy mesons for different states: $1 S$ - blue lines (tall and steep), $1 P$ - orange (short and wide), $2 S$ - green (with steps). Spin structure of the meson does not matter, hence, e.g., $\eta_{b}$ and $\Upsilon$ have the same $F_{1}(x)$ and $F_{2}(x)$.

\section{Conclusion}

The charge radii of heavy mesons and heavy baryons I calculated are in acceptably good agreement with other theoretical results were the comparison is possible. The agreement is especially good with Lattice results, which is surprising because of simplicity of the wave functions I used and the severity of approximations made to obtain those wave functions. The magnetic moments for heavy quarkonia are also in agreement with other results, but the magnetic moments of mixedflavor particles are considerably bigger when compared with results found in literature. I also calculated the structure functions of heavy hadrons as a demonstration of utility of the method. 

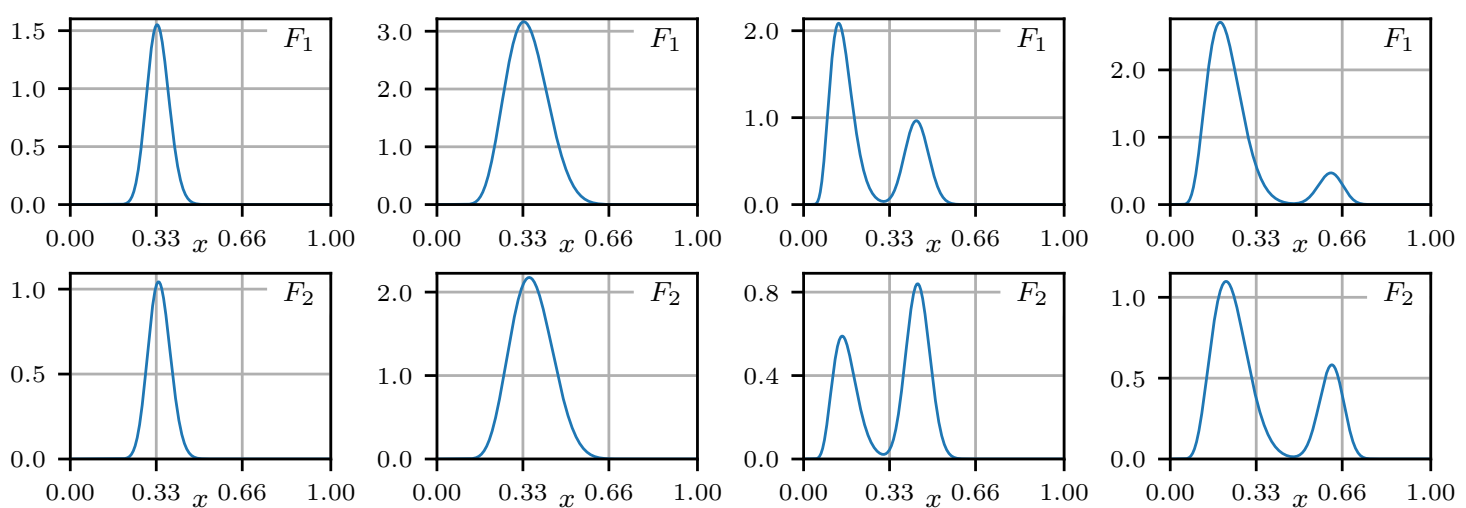

(a) $b b b$

(b) $c c c$

(c) $b b c$

(d) $c c b$

Figure 2: Structure functions of ground states of heavy baryons.

\section{References}

[1] S. D. Głazek, M. Gómez-Rocha, J. More and K. Serafin, Renormalized quark-antiquark Hamiltonian induced by a gluon mass ansatz in heavy-flavor QCD, Phys. Lett. B773 (2017) 172 [1705. 07629 ].

[2] K. Serafin, M. Gómez-Rocha, J. More and S. D. Głazek, Approximate Hamiltonian for baryons in heavy-flavor QCD, Eur. Phys. J. C78 (2018) 964 [1805. 03436 ].

[3] K. Serafin, Bound States of Heavy Quarks in Renormalization Group Procedure for QCD, Ph.D. thesis, University of Warsaw, 2019.

[4] L. Adhikari, Y. Li, M. Li and J. P. Vary, Form factors and generalized parton distributions of heavy quarkonia in basis light front quantization, Phys. Rev. C99 (2019) 035208 [1809. 06475 ].

[5] K. Raya, M. A. Bedolla, J. J. Cobos-Martínez and A. Bashir, Heavy quarkonia in a contact interaction and an algebraic model: mass spectrum, decay constants, charge radii and elastic and transition form factors, Few Body Syst. 59 (2018) 133 [1711. 00383 ].

[6] J. J. Dudek, R. G. Edwards and D. G. Richards, Radiative transitions in charmonium from lattice QCD, Phys. Rev. D73 (2006) 074507 [hep-ph / 0601137 ].

[7] M. S. Bhagwat and P. Maris, Vector meson form factors and their quark-mass dependence, Phys. Rev. C77 (2008) 025203 [nucl-th/ 0612069 ].

[8] K. U. Can, G. Erkol, M. Oka and T. T. Takahashi, Look inside charmed-strange baryons from lattice QCD, Phys. Rev. D92 (2015) 114515 [1508.03048].

[9] T. A. Lahde, Exchange current operators and electromagnetic dipole transitions in heavy quarkonia, Nucl. Phys. A714 (2003) 183 [hep-ph/0208110].

[10] R. Dhir, C. S. Kim and R. C. Verma, Magnetic Moments of Bottom Baryons: Effective mass and Screened Charge, Phys. Rev. D88 (2013) 094002 [1309.4057].

[11] A. Faessler, T. Gutsche, M. A. Ivanov, J. G. Korner, V. E. Lyubovitskij, D. Nicmorus et al., Magnetic moments of heavy baryons in the relativistic three-quark model, Phys. Rev. D73 (2006) 094013 [hep-ph/0602193].

[12] V. Simonis, Improved predictions for magnetic moments and M1 decay widths of heavy hadrons, 1803.01809 .

[13] V. Šimonis, Magnetic properties of ground-state mesons, Eur. Phys. J. A52 (2016) 90 [1604.05894]. 